\title{
Qualidade e eficiência de produtos naturais no tratamento preservativo das madeiras de Araucaria angustifolia, Eucalyptus viminalis e Pinus taeda
}

\author{
Quality and efficiency of natural products in the preservative treatment of the woods of Araucaria \\ angustifolia, Eucalyptus viminalis, and Pinus taeda
}

\author{
Magnos Alan Vivian*, Évelyn Janaina Grosskopf, Gláucia Cota Nunes, Adriana Terumi Itako, Karina \\ Soares Modes
}

Universidade Federal de Santa Catarina, Curitibanos, SC, Brasil. *Autor para correspondência: magnos.alan@ufsc.br.

Submissão: 13/02/2019 | Aceite: 10/12/2019

\begin{abstract}
RESUMO
A madeira, devido sua constituição orgânica, é altamente suscetível a agentes biodeterioradores, tornando-se essencial o tratamento preservativo àquelas que apresentam baixa durabilidade natural. Atualmente, os principais produtos utilizados no tratamento preservativo da madeira são o CCA e o CCB, os quais em razão da sua constituição são tóxicos ao meio ambiente e a saúde humana. Frente à essa situação, torna-se essencial o desenvolvimento de estudos acerca de produtos naturais que apresentem ação biocida, podendo assim ser utilizado no tratamento de madeira, conferindo maior proteção e aumentando a vida útil da peça. Levando em consideração esta perspectiva, o presente estudo teve por objetivo avaliar a qualidade (penetração e retenção) e eficiência de preservantes naturais aplicados no tratamento das madeiras de Araucaria angustifolia, Eucalyptus viminalis e Pinus taeda, submetidas ao apodrecimento acelerado em condições de laboratório. As madeiras das três espécies foram submetidas ao tratamento preservante com tanino, tall oil e óleo de nim, utilizando o método de imersão rápida a frio, na concentração de $5 \%$ do produto preservante, por cinco minutos. Na sequência foram avaliadas através do ensaio de apodrecimento acelerado sob à ação dos fungos apodrecedores Trametes versicolor e Gloeophyllum trabeum, seguindo as recomendações das normas ASTM D 2017 (2005) e ASTM D 1413 (1999). Com base nos resultados, observou-se que a madeira de $P$. taeda exibiu a melhor penetração aos preservantes óleo de nim e tall oil. O tanino retratou penetração vascular para as três espécies. Os melhores valores de retenção foram das madeiras de $A$. angustifolia e $P$. taeda, para todos os tratamentos aplicados. Quanto a eficiência, em comparação aos resultados encontrados para as outras espécies, todos os tratamentos foram eficientes para a madeira de $P$. taeda quando em contato ao fungo de podridão parda (G. trabeum).
\end{abstract}

PALAVRAS-CHAVE: preservação da madeira, apodrecimento acelerado, tanino, óleo de nim, tall oil.

\begin{abstract}
Wood, due to its organic constitution, is highly susceptible to biodeterioration agents, making preservative treatment essential to those with low natural durability. Currently, the main products used in the preservative treatment of wood are CCA and CCB, which because of their constitution, are toxic to the environment and human health. Faced with this situation, it is essential to develop studies on natural products that have a biocidal action and thus can be used in the wood treatment, giving greater protection and increasing the lifespan of the piece. Considering this perspective, the present study aims to assess the quality (penetration and retention) and the efficiency of natural preservatives applied to the treatment of Araucaria angustifolia, Eucalyptus viminalis, and Pinus taeda, subjected to accelerated rotting under laboratory conditions. The woods of the three species were submitted to preservative treatment with tannin, tall oil, and neem oil, using the cold quick immersion method at the concentration of $5 \%$ of the product preservative solution for five minutes. In the sequence, they were assessed by the accelerated rotting test under the action of rotting fungi Trametes versicolor and Gloeophyllum trabeum, following the recommendations of ASTM D 2017 (2005) and ASTM D 1413 (1999). Based on the results, it was observed that $P$. taeda wood showed the best penetration to the neem and tall oil preservatives. The tannin presented vascular penetration for the three species. The best retention values were from $A$. angustifolia and $P$. taeda, for all treatments applied. Regarding the efficiency, comparing the results found
\end{abstract}


for the other species, all treatments were efficient for $P$. taeda wood when in contact with the brown rot fungi (G. trabeum).

KEYWORDS: wood preservation, accelerated decay, tannin, neem oil, tall oil.

\section{INTRODUÇÃO}

A madeira é um material de origem orgânica, sendo altamente suscetível a deterioração por agentes biológicos (fungos, bactérias, insetos e brocas marinhas), os quais podem atacar a celulose, hemicelulose e lignina.

Tendo em vista a perspectiva apresentada, a durabilidade natural da madeira é uma característica de grande importância, pois determina o seu uso. No entanto, as madeiras que apresentam tal característica são, em sua maioria, de espécies tropicais, as quais devido à grande exploração do século passado estão escassas e atualmente são protegidas por lei.

Com isso, o setor de base florestal passou a utilizar madeiras de espécies exóticas e de rápido crescimento, como por exemplo, o Pinus e Eucalyptus, visando suprir a crescente demanda por madeira. Porém, tais espécies caracterizam-se pela baixa durabilidade natural, logo, necessitam da aplicação de um produto preservante, aumentando assim sua resistência e vida útil.

Atualmente, os principais produtos utilizados no tratamento preservativo da madeira são: CCA (Chromated Copper Arsenate ou Arseniato de Cobre Cromatado) e o CCB (Chromated Copper Boric ou Borato de Cobre Cromatado). Esses produtos são altamente eficazes contra a ação de agentes xilófagos e também a intempéries, porém, em razão de sua composição química, apresentam riscos à saúde humana e ao meio ambiente (MACHADO et al. 2006, AHN et al. 2008, DIAS \& BARREIROS 2017).

Dessa forma, torna-se indispensável a realização de pesquisas sobre produtos naturais que apresentem capacidade de proteger a madeira contra agentes biodeterioradores. WANG et al. (2005), MACHADO et al. (2006), PAES et al. (2012) e SOUZA \& DEMENIGHI (2017) pronunciam-se a favor da utilização de produtos naturais como preservantes da madeira, posto que tais compostos são de origem renovável e não trazem prejuízos ao meio ambiente.

Os compostos naturais são constituídos por substâncias que tem ação biocida, as quais podem ser extraídas de diferentes partes das plantas, como da casca, madeira, folhas e sementes. SOUZA \& DEMENIGHI (2017) citam como exemplo o tanino e o óleo de nim como produtos naturais que podem vir a ser utilizados como preservante. Já BOSSARDI (2014) faz menção ao tall oil, como produto alternativo de fonte renovável.

Segundo CARVALHO \& FERREIRA (1990), o óleo de nim tem como princípio ativo uma substância denominada azadirachtina, responsável por gerar toxidez ao agente xilófago. Já o tall oil age reduzindo a absorção de água capilar no alburno e removendo os componentes que são essenciais para o desenvolvimento do agente (HYVÖNEN et al. 2005). Por fim, o tanino é constituído por substâncias antioxidantes, de alto peso molecular, capazes de precipitar proteínas e conferir poder de adstringência, propriedades estas que irão proporcionar proteção vegetal contra patógenos (PROCITROPICOS 2014).

A utilização de produtos naturais que possibilitem aumentar a resistência da madeira aos agentes xilófagos vem sendo estudada por vários pesquisadores da área, porém, o número de trabalhos publicados ainda é consideravelmente baixo, quando comparado aos produtos químicos.

Neste contexto, SINGH \& SINGH (2011) comentam acerca da dificuldade e limitações de se trabalhar com produtos naturais, tendo em vista que há uma série de fatores que impedem o desenvolvimento de estudos relacionados a esta temática. Um dos fatores limitantes citados por estes autores é a ausência de métodos de tratamento mais aprimorados aos compostos naturais. Em razão dessas limitações e também da burocratização legislativa para registar tais compostos como preservantes de madeira, atualmente há apenas um único preservante natural de madeira que tem registro no Instituto Brasileiro do Meio Ambiente e dos Recursos Naturais Renováveis - IBAMA, este utiliza como princípio ativo o tanino.

Para confirmar a eficácia destes produtos no tratamento preservativo da madeira, empregam-se os ensaios biológicos de campo ou em laboratório, associados aos parâmetros de penetração e retenção desses compostos na madeira, que indicam a qualidade do mesmo. Frente a todo contexto apresentado, o presente estudo teve por objetivo avaliar a qualidade e eficiência de produtos naturais (tanino, óleo de nim e tall oil) no tratamento preservativo das madeiras de Araucaria angustifolia, Eucalyptus viminalis e Pinus taeda em ensaio de laboratório sob à ação de fungos apodrecedores. 


\section{MATERIAL E MÉTODOS}

\section{Coleta e preparo do material}

Para o desenvolvimento do presente estudo utilizou-se as madeiras de Araucaria angustifolia (com aproximadamente 30 anos) e Pinus taeda e Eucalyptus viminalis (ambas com 15 anos). Este material foi adquirido na forma de tábuas junto a uma serraria situada no município de Curitibanos, SC, o qual posteriormente foi transportado para o Laboratório de Recursos Florestais, da Universidade Federal de Santa Catarina, para condução das demais etapas.

Foram confeccionados corpos de prova nas dimensões de $2,5 \times 2,5 \times 0,9 \mathrm{~cm}$ sendo a última dimensão no sentido da grã. Primeiramente, esses corpos de prova passaram por um processo de secagem em condição ambiente para que houvesse uma redução inicial do teor de umidade, seguida de secagem em estufa com circulação forçada de ar a uma temperatura de $60{ }^{\circ} \mathrm{C}$, até estabilização de sua massa. $\mathrm{Na}$ sequência, o material foi pesado em balança analítica (precisão de 0,0001 gramas) para registro da massa inicial e logo após, com auxílio de um paquímetro digital, teve as suas dimensões mensuradas.

\section{Densidade aparente}

Para caracterização das madeiras utilizadas, foi calculada a densidade aparente utilizando os mesmos corpos de prova empregados no ensaio de laboratório, com base nos valores de massa e volume (Equação 1) adquiridos após a secagem em estufa (a $60 \stackrel{\circ}{ }$ ), até massa constante, conforme descrito no item anterior.

$$
\mathrm{Da}=\frac{\mathrm{II}}{\mathrm{v}}
$$

Em que: $\mathrm{Da}=$ densidade aparente, $\mathrm{g} / \mathrm{cm}^{3} ; \mathrm{m}=$ massa, $\mathrm{g} ; \mathrm{v}=$ volume, $\mathrm{cm}^{3}$

\section{Tratamento preservante}

Para o tratamento preservativo foram utilizados três diferentes compostos naturais: óleo de nim, tall oil e tanino. Esses compostos foram adquiridos junto a empresas que comercializam tais substâncias.

A aplicação do produto preservante à madeira foi conduzido utilizando o método de imersão rápida a frio, que consiste em deixar a madeira submersa em contato com o produto por um determinado período de tempo. Em virtude a esse aspecto, esta técnica é classificada como um método de tratamento caseiro (ou sem pressão). Com relação ao preparo das soluções preservativas, utilizou-se a concentração de $5 \%$ do ingrediente ativo a ser testado (Tabela 1).

Tabela 1. Composição dos tratamentos aplicados.

Table 1. Composition of the treatments applied.

\begin{tabular}{lllc}
\hline Tratamento & Espécie & Preservante & Concentração aplicada (\%) \\
\hline 1 & Araucaria angustifolia & Testemunha & - \\
2 & Araucaria angustifolia & Tanino & 5 \\
3 & Araucaria angustifolia & Óleo de nim & 5 \\
4 & Araucaria angustifolia & Tall oil & 5 \\
5 & Eucalyptus viminalis & Testemunha & - \\
6 & Eucalyptus viminalis & Tanino & 5 \\
7 & Eucalyptus viminalis & Óleo de nim & 5 \\
8 & Eucalyptus viminalis & Tall oil & 5 \\
9 & Pinus taeda & Testemunha & - \\
10 & Pinus taeda & Tanino & 5 \\
11 & Pinus taeda & Óleo de nim & 5 \\
12 & Pinus taeda & Tall oil & 5 \\
\hline
\end{tabular}

Após o preparo, os corpos de prova foram submersos na solução, onde permaneceram por 5 minutos, tempo necessário para que ocorresse a penetração do produto na madeira. Encerrando o tempo de tratamento, os corpos de prova passaram por um processo de secagem ao ar livre de aproximadamente 2 semanas, para fixação do produto. Na sequência, as amostras foram dispostas em uma estufa de secagem a $60^{\circ} \mathrm{C}$, até peso constante, para na sequência serem conduzidas as avaliações.

Qualidade do tratamento preservante

A qualidade do tratamento com preservantes naturais foi avaliada por meio dos parâmetros de penetração e retenção.

A penetração refere-se à distribuição e profundidade do produto preservante na peça de madeira (MENDES \& ALVES 1988). A penetração pode ser classificada como: nula, vascular, parcial irregular ou 
periférica e total (SALES-CAMPOS et al. 2003).

Para avaliação da penetração foram selecionados aleatoriamente três corpos de prova de cada tratamento e espécie, os quais, após todas as etapas que envolviam o tratamento, foram cortados ao meio (na face transversal) e lixados para realização da análise visual, para posterior classificação, de acordo com a metodologia apresentada por SALES-CAMPOS et al. (2003), conforme a Figura 1.

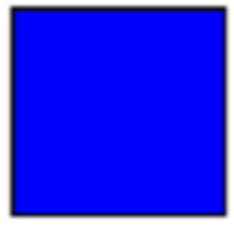

Penetração total

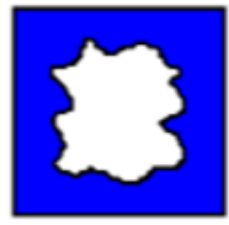

Penetração parcial periférica

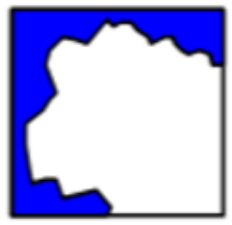

Penetração parcial irregular

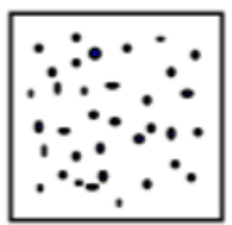

Penetração vascular

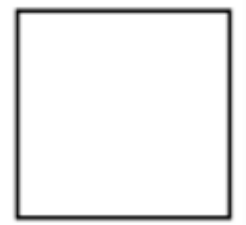

Penetração

nula

Fonte: Adaptado de SALES-CAMPOS et al. (2003).

Figura 1. Padrões utilizados para análise da penetração do produto preservante.

Figure 1. Standards used for the analysis of preservative product penetration.

Já a retenção pode ser definida como a quantidade de produto que ficou retido na madeira, sendo expressa pela relação da massa de produto por unidade de madeira tratada, indicada em quilograma por metro cúbico $\left(\mathrm{kg} / \mathrm{m}^{3}\right)$. A retenção foi calculada conforme a Equação 2.

$$
\mathrm{R}=\frac{(\mathrm{Mf}-\mathrm{Mi})}{\mathrm{V}}
$$

Em que: $\mathrm{R}=$ retenção, $\mathrm{kg} / \mathrm{m}^{3} ; \mathrm{Mf}=$ massa final após tratamento e secagem em estufa a $60 \stackrel{\circ}{\circ} \mathrm{C}, \mathrm{kg} ; \mathrm{Mi}$ $=$ massa inicial (antes do tratamento) após a secagem em estufa a $60{ }^{\circ} \mathrm{C}, \mathrm{kg} ; \mathrm{V}=$ volume, $\mathrm{m}^{3}$.

\section{Eficiência do tratamento preservante}

O ensaio de apodrecimento acelerado foi executado no Laboratório de Fitopatologia, pertencente a Universidade Federal de Santa Catarina. Este ensaio seguiu as metodologias estabelecidas nas normas da American Society for Testing and Materials: ASTM D 2017 (ASTM 2005) e ASTM D 1413 (ASTM 1999), sendo esta última responsável apenas pelas especificações acerca do tipo e umidade do solo a ser utilizado.

Foram empregadas duas espécies de fungos xilófagos provenientes da coleção do Laboratório de Produtos Florestais (LPF), do Serviço Florestal Brasileiro (SFB) de Brasília: Trametes versicolor (Linnaeus ex Fries) Pilat, espécie causadora de podridão branca e Gloeophyllum trabeum (Persoon. ex Fries) Murr., espécie causadora de podridão parda.

Para a preservação dos fungos utilizou-se a metodologia de CASTELLANI (1964), a qual prevê a conservação em água destilada e temperatura ambiente. Tendo em vista que estes microrganismos necessitam de um ambiente e condições nutritivas que favoreçam seu desenvolvimento, foi preparado o meio de cultura BDA (Batata-Dextrose-Ágar), o qual tem sua eficácia cientificamente comprovada ao desenvolvimento de fungos.

Inicialmente, foram preparadas 10 placas de Petri contendo o meio de cultura (5 para cada agente), as quais receberam um pequeno inóculo do micélio de fungo, que após o desenvolvimento passaram a ser chamadas de placas mãe. Em seguida, o material permaneceu em incubadora com fotoperíodo de 12 horas e temperatura de $25{ }^{\circ} \mathrm{C}$ por um período de 15 dias para que houvesse o desenvolvimento do micélio fúngico.

Com o fungo em ótimo estágio de desenvolvimento nessas placas, foi realizado uma nova repicagem, totalizando 25 placas para cada agente, seguido do processo de acondicionamento. Essas placas foram fontes de inóculo para a execução do ensaio de apodrecimento acelerado. Cabe ressaltar que todos os materiais utilizados, incluindo o meio de cultura, passaram por um processo de esterilização $\left(121^{\circ} \mathrm{C}\right.$ a 20 minutos) em autoclave antes da repicagem dos fungos.

Para condução do ensaio utilizaram-se frascos de vidro transparente com capacidade de $600 \mathrm{~mL}$, com tampa rosqueável, os quais foram preenchidos com $100 \mathrm{~g}$ de solo horizonte $\mathrm{B}, \mathrm{com} \mathrm{pH} 6,0$, isento de matéria orgânica. Após a determinação do teor de umidade do solo, a capacidade de campo foi ajustada para 130\%, com adição de água destilada, segundo as recomendações da norma ASTM D 1413 (ASTM 1999). 
Para o desenvolvimento inicial do fungo, foi depositado no interior dos frascos, uma placa suporte medindo $0,3 \times 3,0 \times 3,0 \mathrm{~cm}$ (radial, tangencial e longitudinal) respectivamente, de acordo com a Figura 2 . As placas suporte foram confeccionadas com madeira de espécies distintas para cada fungo, sendo que $0 T$. versicolor teve seu estabelecimento inicial em placas de Eucalyptus sp. e o G. trabeum em placas de Pinus sp. Após este procedimento, os frascos, parcialmente fechados, passaram por um processo de esterilização em autoclave com temperatura de $121^{\circ} \mathrm{C}$ por cerca de 45 minutos.

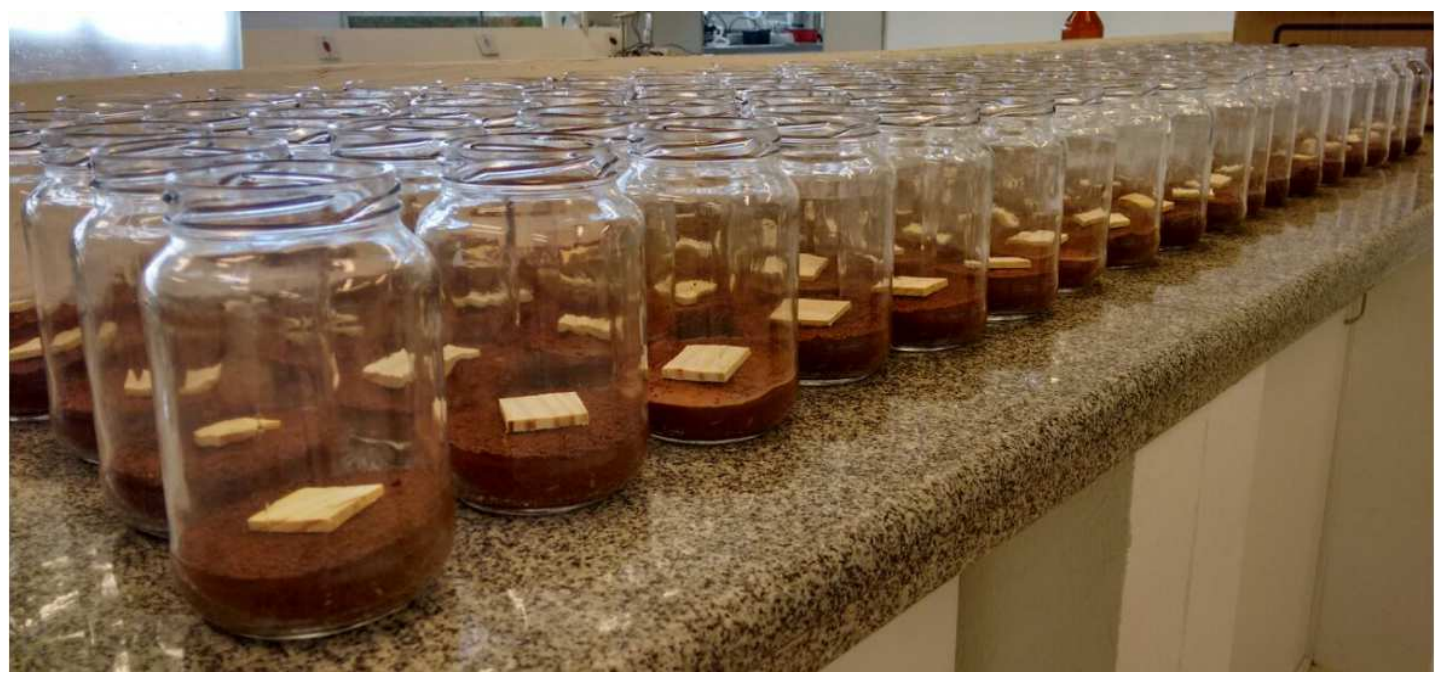

Figura 2. Frascos de ensaio contendo placa suporte para o desenvolvimento dos fungos.

Figure 2. Test flasks containing support plate for the fungi development.

Após resfriamento, em cada frasco, sobre a placa suporte, foram depositados cinco discos de micélio do fungo a ser avaliado, os quais permaneceram na incubadora por 3 semanas para desenvolvimento sobre a placa (Figura 3A). Após este período, introduziu-se um corpo de prova (que também passou pelo processo de esterilização) em cada frasco (Figura 3B e C), que permaneceram na incubadora de crescimento por 16 semanas em contato com o fungo.

Encerrado o período de apodrecimento, os corpos de prova foram retirados dos frascos de vidro e submetidos a uma limpeza cuidadosa, que com o auxílio de uma escova de cerdas macias foi removido todo excesso de micélio aderido (Figura 3DEF). Posteriormente, foram acondicionados em estufa, sob as mesmas condições pré-ensaio $\left(60^{\circ} \mathrm{C}\right)$ até obter peso constante e foram novamente pesados para determinação da massa final.

A eficiência dos preservantes naturais quanto a inibição do ataque dos fungos apodrecedores foi avaliada a partir da perda da massa dos corpos de prova, por meio da diferença dos valores de massa inicial e massa final, conforme Equação 3.

$$
\mathrm{FN}_{\mathrm{cp}}=\frac{\mathrm{Mi}-\mathrm{Mf}}{\mathrm{Mi}} \approx 100
$$

Em que: $\mathrm{PM}_{\mathrm{cp}}=$ perda de massa do corpo de prova, \%; $\mathrm{Mi}=$ massa inicial após a secagem em estufa $\left(60^{\circ} \mathrm{C}\right), \mathrm{g} ; \mathrm{Mf}=$ massa final após a secagem em estufa $\left(60^{\circ} \mathrm{C}\right), \mathrm{g}$.

A partir dos valores médios de perda de massa a resistência da madeira foi classificada de acordo com a norma ASTM D 2017 (ASTM 2005) (Tabela 2).

Tabela 2. Classe de resistência da madeira ao ataque de fungos apodrecedores.

Table 2. Class of resistance of the wood to the attack of rotting fungi.

\begin{tabular}{lc}
\hline Classes de Referência & Perda de massa (\%) \\
\hline Altamente Resistente (AR) & $1-10$ \\
Resistente (R) & $11-24$ \\
Moderadamente Resistente (MR) & $24-44$ \\
Não Resistente (NR) & $>45$ \\
\hline
\end{tabular}

Fonte: ASTM 2005. 

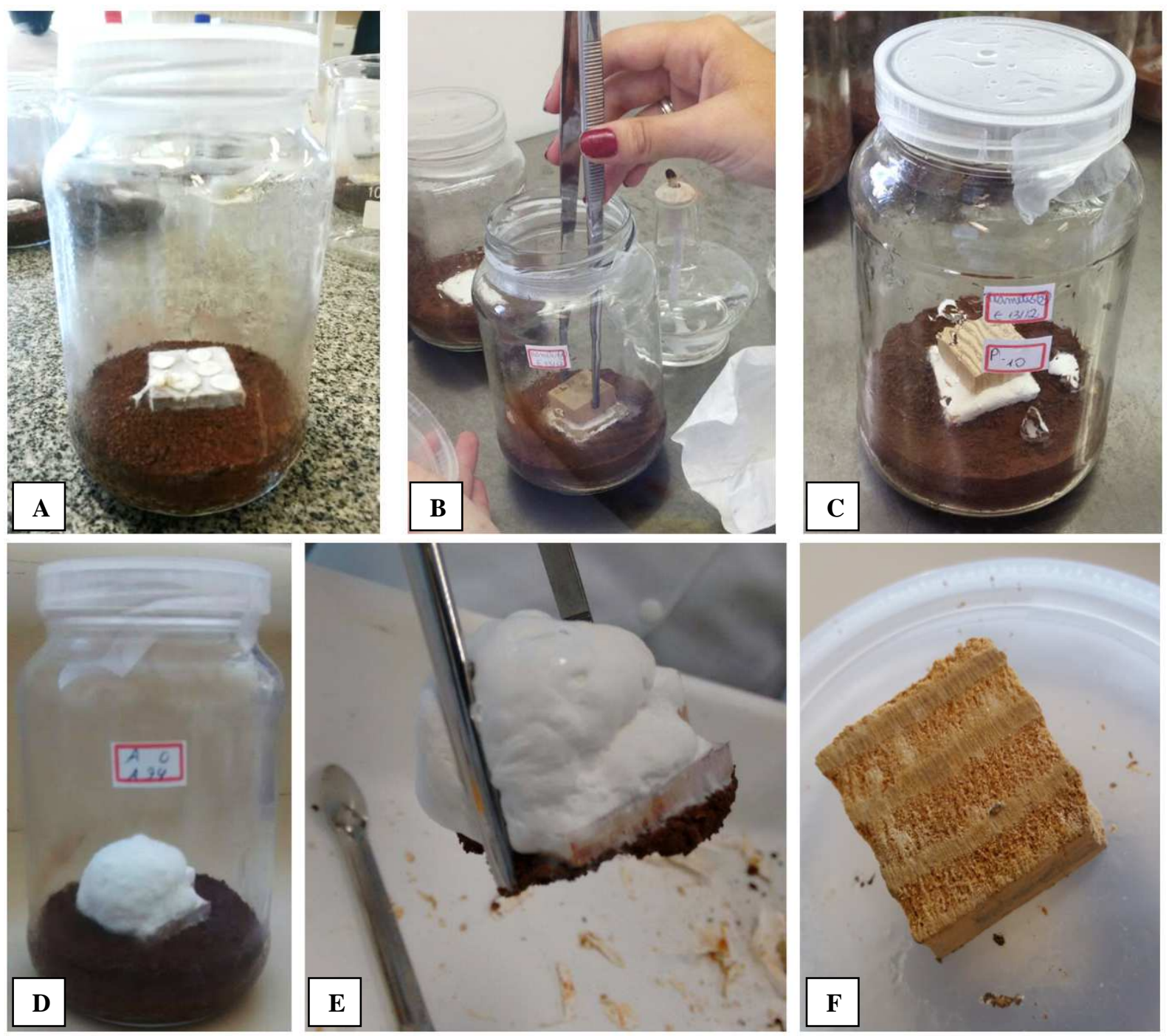

Figura 3. Placa suporte recoberta com o micélio do fungo $T$. versicolor $(A)$, deposição do corpo de prova sobre a placa suporte $(B$ e $C$ ). Etapa final do ensaio de apodrecimento acelerado: micélio do fungo T. versicolor recobrindo tanto a placa suporte quanto o corpo de prova (D); Remoção do material do corpo de prova para limpeza $(E)$; Corpo de prova após a retirada do micélio do fungo $(F)$.

Figure 3. Support plate covered with the mycelium of the fungus $T$. versicolor $(A)$, deposition of the specimen on the support plate ( $B$ and $C$ ). Final step of the accelerated rotting test: mycelium of the fungus $T$. versicolor covering both the support plate and the specimen (D); Removal of test specimen material for cleaning $(E)$; Test specimen after removal of fungal mycelium $(F)$.

\section{Análise estatística}

Os dados referentes ao presente estudo foram processados e analisados de forma eletrônica a partir do software estatístico R 3.4.2 em conjunto com o complemento "R-studio", que atenderam aos objetivos da pesquisa.

A retenção do produto preservante foi analisada em delineamento inteiramente casualizado (DIC), em arranjo fatorial $3 \times 3$, sendo os fatores: espécie (3 níveis) e produto preservante (3 níveis).

O ensaio de apodrecimento acelerado foi realizado em delineamento inteiramente casualizado (DIC), em arranjo tri-fatorial, com os seguintes fatores: espécies (3 níveis), tratamentos preservantes (4 níveis) e fungos (2 níveis), um total de 12 tratamentos. Este ensaio em questão foi composto por: duas espécies de fungos apodrecedores (podridão parda e branca), com 7 repetições por fungo, para cada tratamento (Testemunha, Tanino, Óleo de nim e Tall oil), e espécies (Araucaria angustifolia, Eucalyptus viminalis e Pinus taeda), totalizando 168 corpos de prova.

A fim de assegurar a normalidade dos resultados da análise de variância foi realizado o teste de Shapiro-Wilk.

Todos os dados foram avaliados por meio de análise de variância (ANOVA), com posterior comparação de média pelo teste de Tukey, ao nível de $5 \%$ de probabilidade de erro. 


\section{RESULTADOS E DISCUSSÃO \\ Densidade aparente}

A densidade aparente das madeiras de $A$. angustifolia, E. viminalis e $P$. taeda seca em estufa a $60{ }^{\circ} \mathrm{C}$, antes de receberem o tratamento preservativo, pode ser observada na Tabela 3. A respeito disso, verificou-se pelo teste de médias que houve diferença estatística entre as espécies, sendo a madeira de $E$. viminalis mais densa, seguida da madeira de $A$. angustifolia e $P$. taeda.

Tabela 3. Densidade aparente das espécies empregadas no estudo.

Table 3. Apparent density of the species used in the study.

\begin{tabular}{ll}
\hline Espécie & Densidade aparente $\left(\mathrm{g} / \mathrm{cm}^{3}\right)$ \\
\hline Araucaria angustifolia & $0,534 \mathrm{~b}$ \\
Eucalyptus viminalis & $0,748 \mathrm{a}$ \\
Pinus taeda & $0,427 \mathrm{c}$ \\
\hline
\end{tabular}

Médias seguidas da mesma letra, não apresentam diferença estatística significativa através do teste de médias (Tukey, $p>0,05)$.

Com base na classificação proposta por DURLO (1991), as madeiras de P. taeda, A. angustifolia e $E$. viminalis foram classificadas como de densidade leve, média e pesada, respectivamente.

A $A$. angustifolia exibiu densidade média de $0,534 \mathrm{~g} / \mathrm{cm}^{3}$. FRIGERI et al. (2017), determinaram a densidade aparente das madeiras de $A$. angustifolia a $12 \%$ de umidade e observaram uma densidade de $0,400 \mathrm{~g} / \mathrm{cm}^{3}$, inferior ao valor encontrado neste estudo.

BELTRAME et al. (2010) desenvolveram um estudo acerca das propriedades físicas e mecânicas da madeira de $A$. angustifolia em três diferentes estratos de floresta (superior, médio e inferior). Dentre as características avaliadas, esses autores verificaram que a densidade aparente ( $12 \%$ de umidade) foi maior para madeira do estrato superior $\left(0,512 \mathrm{~g} / \mathrm{cm}^{3}\right)$, valor este muito semelhante ao encontrado no presente estudo.

Observa-se que a madeira de E. viminalis é mais densa do que as demais espécies empregadas neste estudo, uma vez que o valor é de $0,748 \mathrm{~g} / \mathrm{cm}^{3}$. IWAKIRI et al. (2013) avaliaram o potencial de 9 espécies de Eucalyptus, com idade variando entre 18 a 21 anos, e observaram para madeira de $E$. viminalis densidade aparente de $0,617 \mathrm{~g} / \mathrm{cm}^{3}$.

Em análise as características físicas da madeira de três espécies do gênero Eucalyptus com 18 anos, LOPES (2007) verificou densidade aparente de $0,780 \mathrm{~g} / \mathrm{cm}^{3}$ para $E$. dunnii, $0,880 \mathrm{~g} / \mathrm{cm}^{3}$ para E. urophylla e $0,750 \mathrm{~g} / \mathrm{cm}^{3}$ para $E$. grandis. Tais valores são semelhantes ao observado neste estudo para espécie de $E$. viminalis $\left(0,748 \mathrm{~g} / \mathrm{cm}^{3}\right)$.

STURION et al. (1988), estudando as propriedades da madeira de E. viminalis para fins energéticos verificaram que a madeira aos 4 anos de idade apresenta densidade aparente de $0,490 \mathrm{~g} / \mathrm{cm}^{3}$, já a madeira com 7 anos apresenta $0,520 \mathrm{~g} / \mathrm{cm}^{3}$. Ambos os valores encontrados são inferiores aos apresentados na Tabela 4, o que já era de se esperar, tendo em vista a grande diferença de idade.

A madeira de $P$. taeda apresentou densidade aparente de $0,427 \mathrm{~g} / \mathrm{cm}^{3}$, valor semelhante ao encontrado por KLOCK (2000) $\left(0,419 \mathrm{~g} / \mathrm{cm}^{3}\right)$ e inferior ao encontrado por MELCHIORETTO \& ELEOTÉRIO (2003) com 25 anos.

BALLARIN \& PALMA (2003) avaliaram a diferença de densidade aparente (12\% de umidade) entre a madeira juvenil e adulta de $P$. taeda com 37 anos. Em função da idade, os resultados encontrados por esses autores diferiram aos apresentados neste estudo, sendo $0,674 \mathrm{~g} / \mathrm{cm}^{3}$ para madeira adulta e 0,536 $\mathrm{g} / \mathrm{cm}^{3}$ para juvenil.

De acordo com ROLIM \& FERREIRA (1974), a densidade da madeira aumenta gradativamente em função da idade, sendo que a estabilização desse valor varia de espécie para espécie. Situação que vem a justificar a diferença de densidade das duas coníferas empregas neste estudo.

\section{Qualidade do tratamento}

Os resultados da penetração do tanino, óleo de nim e tall oil nos corpos de prova podem ser observados na Tabela 4. Nota-se que, independente do produto aplicado, nenhum dos corpos de prova apresentou penetração nula.

Em análise aos resultados apresentados, observa-se que a madeira de $A$. angustifolia apresentou penetração parcial periférica quando tratada com óleo de nim e irregular para o tall oil. Já o $E$. viminalis exibiu penetração irregular a estes dois produtos citados, em contrapartida, o $P$. taeda retratou penetração total. Diferentemente dos outros tratamentos, o tanino manifestou comportamento igual para todas as 
espécies, retratando penetração vascular.

Tabela 4. Resultados da análise de penetração da face interna dos corpos de prova.

Table 4. Results of the penetration analysis of the internal face of the specimens.

\begin{tabular}{lccl}
\hline Tratamento & & Espécie - Tipo de penetração & \\
\hline Tanino & Araucaria angustifolia & Eucalyptus viminalis & Pinus taeda \\
Óleo de nim & Vascular & Vascular & Vascular \\
Tall oil & Parcial periférica & Parcial irregular & Total \\
\hline
\end{tabular}

A madeira de E. viminalis apresentou penetrações consideravelmente baixas em todos os tratamentos aplicados. Uma justificativa para tal resultados deve-se a alta densidade da madeira, o que implica em menor porosidade e, consequentemente, menor permeabilidade, tendo em vista que os poros estão devidamente ocupados por outras substâncias que constituem a madeira.

Além disso, SALES-CAMPOS et al. (2003) aponta que as madeiras do gênero Eucalyptus, assim como as demais folhosas, caracterizam-se anatomicamente pela presença de tiloses, as quais durante o desenvolvimento anual e formação do cerne irão ocasionar a obstrução dos vasos e, consequentemente, a má distribuição do produto preservante. Corroborando essa informação, MORESCHI (2014) descreve que as tiloses são responsáveis por impedir a penetração do produto no sentido longitudinal, pelo qual o produto teria acesso aos tecidos vizinhos.

VIVIAN et al. (2012) em estudo realizado com as madeiras de Eucalyptus grandis e Eucalyptus cloeziana, ambas com 16 anos de idade, tratadas em autoclave com CCA, observaram a penetração vascular para madeira de E. grandis e penetração parcial irregular nas de E. cloeziana. Os autores explicam que esse resultado é decorrente da idade das árvores, considerando que na idade de 16 anos apresentam maior proporção de cerne, sendo este mais impermeável a solução preservante.

Com relação a penetração do preservante nas madeiras de coníferas, observa-se (Tabela 4) que o $P$. taeda exibiu resultados mais satisfatórios, denotando penetração total aos compostos tall oil e óleo de nim. Muito embora apresentem características bastante semelhantes, a madeira de $P$. taeda é descrita como de menor densidade (Tabela 3), logo, a permeabilidade será maior, possibilitando assim a passagem mais facilmente do produto pelos canais resiníferos.

Nessa mesma linha de considerações, MORESCHI (2014) menciona a inexistência de canais resiníferos na madeira de $A$. angustifolia, com isso, a penetração do produto irá ocorrer exclusivamente pelos traqueídeos e pelas pontuações. Ainda, esse mesmo autor aponta que o tratamento de madeiras que possuem essa característica é mais uniforme. Tal situação vem a justificar a diferença de penetração verificada entre as madeiras de coníferas utilizadas quando tratadas com óleo de nim e tall oil.

No que tange os fatores que interferem na penetração do produto na madeira, MORESCHI (2014) elenca a profundidade da madeira de alburno, os sentidos anatômicos da madeira, vasos com tiloses, canais resiníferos e também os tipos de pontuações.

Uma das dificuldades encontradas para análise da retenção é o fato de que não existe produto que possa ser aplicado para reagir com estes preservantes como ocorre no caso dos hidrossolúveis como o CCA e CCB, em que se emprega a solução de cromoazurol-S, que reage com o cobre das formulações, destacando em cor azulada onde o mesmo penetrou e se distribuiu.

$\mathrm{Na}$ Tabela 5 pode ser observada a retenção calculada para os tratamentos com tanino, óleo de nim e tall oil após a estabilização da massa seca em estufa das madeiras utilizadas.

Em síntese, conforme exposto na Tabela 5, o óleo de nim apresentou os mais satisfatórios valores de retenção para todas as espécies, diferindo estatisticamente dos demais produtos avaliados. PAES et al. (2012) avaliaram a eficiência do óleo de nim e de mamona no tratamento preservativo da madeira de Samaúma (Ceiba pentandra), pelo método de imersão a frio e concluíram que, nas retenções de 10 a 16 $\mathrm{kg} / \mathrm{m}^{3}$, ambos os compostos não foram efetivos no tratamento da madeira.

O tall oil exibiu menor retenção para a madeira de $E$. viminalis $\left(4,93 \mathrm{~kg} / \mathrm{m}^{3}\right)$, que não diferiu da $A$. angustifolia $\left(8,77 \mathrm{~kg} / \mathrm{m}^{3}\right)$, logo, ambas apresentaram diferença estatística apenas a espécie de $P$. taeda que apresentou retenção de $\left(15,81 \mathrm{~kg} / \mathrm{m}^{3}\right)$. A baixa retenção apresentada por esse composto em questão pode ser justificada pela exsudação que o mesmo exibiu para na madeira de $E$. viminalis no decorrer do ensaio. PANOV \& TERZIEV (2010) comentam que a exsudação de compostos oleosos ocorre pelo fato dos mesmos não apresentarem ligação aos elementos estruturais da parede celular, ou ainda, a ausência de oxigênio faz com que o óleo não seja polimerizado, forçando assim sua saída a superfície, o que é 
reforçado pela menor porosidade dessa madeira, sendo equivalente entre as coníferas.

Tabela 5. Retenção média do tanino, óleo de nim e tall oil para as madeiras após serem submetidas ao tratamento por imersão.

Table 5. Average retention of tannin, neem oil, and tall oil for the woods after being submitted to the immersion treatment.

\begin{tabular}{lccc}
\hline Tratamento & \multicolumn{3}{c}{ Espécie - Retenção $\left(\mathrm{kg} / \mathrm{m}^{3}\right)$} \\
\hline Tanino & Araucaria angustifolia & Eucalyptus viminalis & Pinus taeda \\
Óleo de nim & $9,15 \mathrm{bA}$ & $2,18 \mathrm{bB}$ & $8,95 \mathrm{cA}$ \\
Tall oil & $26,67 \mathrm{aB}$ & $9,77 \mathrm{aC}$ & $47,18 \mathrm{aA}$ \\
\hline
\end{tabular}

As médias na vertical seguidas por uma mesma letra minúscula, ou na horizontal, por uma mesma letra maiúscula, não diferem estatisticamente (Tukey $p>0,05)$.

Em comparação com os demais produtos testados, o tanino apresentou a menor retenção para a madeira de E. viminalis $\left(2,18 \mathrm{~kg} / \mathrm{m}^{3}\right)$. De acordo com SINGH \& SINGH (2011) a grande problemática relacionada ao tanino é referente a má fixação na madeira após o tratamento, necessitando assim do uso de aditivos que atuem na imobilização do mesmo na madeira, fato que justifica a menor retenção observava de maneira geral.

Observa-se que as maiores retenções são para a espécie de $P$. taeda, diferindo estatisticamente das demais espécies, com exceção aquela tratada com tanino. Uma possível justificativa que pode ser considerada para tal situação pode estar relacionada a baixa densidade dessa madeira $\left(0,427 \mathrm{~g} / \mathrm{cm}^{3}\right.$, Tabela 3).

Muito embora a retenção expresse a quantidade de produto em quilograma $(\mathrm{kg})$ por metro cúbico $\left(\mathrm{m}^{3}\right)$ de madeira, nem sempre as retenções superiores irão denotar qualidade e eficiência. PANOV \& TERZIEV (2010) mencionam que tal situação poderá alterar a aparência da peça, tendo em vista que o produto em excesso pode exsudar da madeira, além do mais a madeira ficará mais pesada tornando todo o processo mais oneroso.

AMARAL (2012) aponta que a penetração e retenção são propriedades independentes, ou seja, uma penetração total, como é no caso do $P$. taeda, não necessariamente irá implicar em uma maior retenção, ou ainda, aquela que se diz adequada. Logo, não se deve realizar análise comparativa entre estas duas variáveis.

\section{Eficiência do tratamento}

$\mathrm{Na}$ Tabela 6 constam as médias referentes a perda de massa das madeiras de A. angustifolia, $E$. viminalis e $P$. taeda tratadas com preservantes naturais após 16 semanas de ensaio sob a ação dos fungos apodrecedores $G$. trabeum e T. versicolor.

Tabela 6. Percentual de perda de massa (\%) e classificação conforme a norma ASTM D 2017 (ASTM 2005). Table 6. Percentage of mass loss (\%) and classification according to the ASTM D 2017 standard (ASTM 2005).

\begin{tabular}{cccc}
\hline \multirow{2}{*}{ Espécie } & \multirow{2}{*}{ Tratamento } & \multicolumn{2}{c}{ Fungo } \\
\cline { 3 - 4 } & & G. trabeum & T. versicolor \\
\cline { 2 - 4 } Araucaria angustifolia & Testemunha & $27,98 \mathrm{MR}$ & $21,00 \mathrm{R}$ \\
\cline { 2 - 4 } & Tanino & $27,90 \mathrm{MR}$ & $20,81 \mathrm{R}$ \\
& Tall oil & $28,40 \mathrm{MR}$ & $22,21 \mathrm{R}$ \\
& Óleo de nim & $28,87 \mathrm{MR}$ & $19,34 \mathrm{R}$ \\
\hline \multirow{4}{*}{ Eucalyptus viminalis } & Testemunha & $11,20 \mathrm{R}$ & $28,30 \mathrm{MR}$ \\
& Tanino & $12,08 \mathrm{R}$ & $21,05 \mathrm{MR}$ \\
& Tall oil & $11,82 \mathrm{R}$ & $22,52 \mathrm{MR}$ \\
& Óleo de nim & $8,55 \mathrm{AR}$ & $21,66 \mathrm{MR}$ \\
\hline \multirow{2}{*}{ Pinus taeda } & Testemunha & $41,77 \mathrm{MR}$ & $24,39 \mathrm{MR}$ \\
& Tanino & $30,98 \mathrm{MR}$ & $30,30 \mathrm{MR}$ \\
& Tall oil & $29,18 \mathrm{MR}$ & $24,69 \mathrm{MR}$ \\
& Óleo de nim & $32,87 \mathrm{MR}$ & $26,79 \mathrm{MR}$ \\
\hline
\end{tabular}

Em que: $A R$ = altamente resistente; $R$ = resistente; $M R$ = moderadamente resistente. 
Em análise aos resultados apresentados, verifica-se que em geral a perda de massa causada por ambos os fungos foi semelhante para as três espécies empregadas no experimento. Entretanto, é evidente que, independente do tratamento, o fungo de podridão parda ( $G$. trabeum) deteriorou mais profundamente as madeiras de $A$. angustifolia e $P$. taeda, classificando ambas como moderadamente resistentes.

Uma possível justificativa para situação apresentada é citada no estudo desenvolvido por SOARES (1998), que comenta sobre a preferência alimentar dos fungos de podridão parda por madeiras de coníferas. Corroborando essa informação, MARTíNEZ et al. (2005) destacaram que tal ação está amplamente relacionada a densidade da madeira, tendo em vista que em geral as madeiras do grupo citado são mais macias, o que vem a facilitar a deterioração por estes microrganismos.

No que se refere a madeira de $P$. taeda em relação ao fungo $G$. trabeum, observa-se que, independente do produto aplicado, houve menor perda de massa, em comparação a testemunha, porém ambas foram classificadas como moderadamente resistente. Para o fungo $T$. versicolor a classificação foi integralmente a mesma, porém, os valores de perda de massa foram superiores aos da testemunha. $A$ mesma situação é vista para a madeira de Araucária quando submetida as mesmas condições.

Em contrapartida, a madeira de $A$. angustifolia foi classificada como resistente ao fungo de podridão branca ( $T$. versicolor), independente do tratamento.

Por fim, mas não menos importante, a madeira de $E$. viminalis quando exposta ao fungo de podridão branca ( $T$. versicolor) foi classificada como moderadamente resistente a todos os tratamentos. Já ao $G$. trabeum, a deterioração causada foi consideravelmente baixa em todos os tratamentos, logo, foi considerada resistente, com exceção ao tratamento óleo de nim que foi altamente resistente.

As interações fatoriais entre as espécies e preservantes, seguido do teste de médias (Tukey), estão apresentadas na Tabela 7.

Tabela 7. Comparação múltipla de médias para o percentual de perda de massa (\%), em função da interação dos fatores: espécies, fungos e preservantes.

Table 7. Multiple comparison of means for the percentage of mass loss (\%), as a function of the interaction of the factors: species, fungi and preservatives.

\begin{tabular}{lllll}
\hline \multirow{2}{*}{ Espécie } & \multicolumn{4}{c}{ Trametes versicolor } \\
\cline { 2 - 5 } & Testemunha & Tanino & Óleo de nim & Tall oil \\
\cline { 2 - 5 } A. angustifolia & $21,00 \mathrm{aA}$ & $20,81 \mathrm{bA}$ & $19,34 \mathrm{aA}$ & $22,21 \mathrm{aA}$ \\
E. viminalis & $28,30 \mathrm{aA}$ & $21,05 \mathrm{bA}$ & $21,66 \mathrm{aA}$ & $22,52 \mathrm{aA}$ \\
P. taeda & $24,39 \mathrm{aA}$ & $30,30 \mathrm{aA}$ & $26,79 \mathrm{aA}$ & $24,69 \mathrm{aA}$ \\
\hline & \multicolumn{4}{c}{ Gloeophyllum trabeum } \\
\hline A. angustifolia & $27,98 \mathrm{bA}$ & $27,90 \mathrm{aA}$ & $26,87 \mathrm{aA}$ & $28,40 \mathrm{aA}$ \\
E. viminalis & $11,20 \mathrm{cA}$ & $12,08 \mathrm{aA}$ & $8,55 \mathrm{aA}$ & $11,82 \mathrm{aA}$ \\
P. taeda & $41,77 \mathrm{aA}$ & $30,98 \mathrm{aB}$ & $32,87 \mathrm{aB}$ & $29,18 \mathrm{aB}$ \\
\hline
\end{tabular}

As médias na vertical seguidas por uma mesma letra minúscula não diferem estatisticamente. As médias na horizontal seguidas por uma mesma letra maiúscula não diferem estatisticamente.

Com base nos resultados apresentados, verifica-se que óleo de nim retratou mais efetividade a madeira de $A$. angustifolia, sob à ação dos dois fungos apodrecedores empregados neste estudo, porém, para ambas as situações, não houve diferença estatística com relação aos outros tratamentos.

Neste mesmo contexto, observa-se que para a mesma espécie ( $A$. angustifolia) o composto tall oil foi ineficiente no combate destes agentes, pois teve efeito contrário do esperado, fazendo com que a madeira apresentasse valor de perda de massa superior àquela que não recebeu tratamento.

Por fim, com relação a deterioração dos dois fungos apodrecedores, o tanino teve o mesmo comportamento para madeira de $A$. angustifolia, reduzindo um pouco o percentual de perda de massa em comparação a testemunha, porém, estatisticamente apresentou o mesmo comportamento.

Doutro norte, a deterioração na madeira de E. viminalis foi maior quando submetida ao ataque do fungo de podridão branca ( $T$. versicolor), pois estes agentes atacam preferencialmente as madeiras pertencentes ao grupo das angiospermas. Mesmo apresentando uma deterioração mais efetiva, todos os tratamentos aplicados mostraram-se eficazes na redução do percentual de perda de massa em comparação a madeira não tratada (testemunha), contudo, não houve diferença estatística entre todos os tratamentos.

Em contrapartida, sob a ação do fungo de podridão parda, a madeira de E. viminalis teve um aumento 
de perda de massa quando tratada com tanino e tall oil em relação a testemunha. Logo, pode-se pressupor que, mesmo estando na mesma classe de resistência, o preservante óleo de nim foi, numericamente, mais eficiente. Para ambas as situações apresentadas a espécie retratou estatisticamente 0 mesmo comportamento para todos os tratamentos, incluindo a testemunha.

A madeira de $P$. taeda apresentou valores de perda de massa superiores para o fungo $T$. versicolor, independente do tratamento aplicado, ou seja, nenhum dos produtos testados mostrou-se eficaz ao combate deste microrganismo, não havendo diferença estatística. Além disso, os tratamentos proporcionaram um aumento no percentual de perda de massa em comparação a madeira que não recebeu tratamento (testemunha).

Ainda, para esta mesma espécie ( $P$. taeda), ao fungo G. trabeum, a maior perda de massa foi para a madeira que não recebeu tratamento (testemunha $=41,77 \%$ ), diferindo estatisticamente daquelas que receberam o tratamento preservante. Neste contexto, observa-se que os tratamentos preservativos reduziram de forma significativa a perda de massa referente ao fungo de podridão parda (G. trabeum).

PANOV \& TERZIEV (2010) avaliaram a resistência de uma madeira de conífera, não tratada e tratada com éster de tall oil, sob à ação dos fungos de podridão branca ( $T$. versicolor) e parda (G. trabeum), em ensaio de laboratório. Como resultado, esses autores observaram que ao fungo $T$. versicolor a perda de massa foi de 19,3\% para madeira não tratada e 6,8\% para aquela que recebeu o tratamento e para o fungo G. trabeum foi de $28,5 \%$ e $8,9 \%$, respectivamente. Ao exposto, concluíram que o produto avaliado é eficiente, aumentando consideravelmente a resistência da madeira.

TEMIZ et al. (2008) realizaram um estudo acerca da resistência à deterioração da madeira de Pinus sylvestris tratado com ácido bórico e derivados de tall oil, separadamente ou em combinação, sob ação de dois fungos de podridão parda (Postia placenta e Coniophora puteana). Como resultados esses autores verificaram que apenas o tall oil com $90 \%$ de ácido resínico livre combinado a $2 \%$ de ácido bórico foi efetivo no combate dos fungos, tendo em vista que exibiram perda de massa inferior a $3 \%$.

No estudo realizado por TEIXEIRA et al. (2015), os pesquisadores testaram a eficiência do óleo de nim em diferentes concentrações (5 e 50\%) no tratamento preservativo da madeira de Pinus caribaea sobre ação do fungo apodrecedor $T$. versicolor. Os resultados obtidos foram mais satisfatórios para a concentração de $50 \%$, ou seja, proporcionou um aumento significativo na proteção à deterioração da madeira. Já para a concentração de 5\%, a mesma utilizada no presente estudo, foram menos efetivos ao objetivo da pesquisa.

Uma alternativa para melhorar a eficácia dos produtos naturais como preservantes de madeira é fazer uso de aditivos, com o intuito de aumentar a penetração e fixação do produto na parede celular. Para TEMIS et al. (2008), uma boa maneira de melhorar a efetividade dos produtos naturais como preservante de madeira é adicionar junto ao composto determinadas concentrações de ácido bórico.

\section{CONCLUSÃO}

As madeiras de A. angustifolia e E. viminalis apresentaram penetração consideravelmente baixa. Já o $P$. taeda exibiu penetração total ao tall oil e óleo de nim, visto que é uma madeira menos densa e mais permeável. As madeiras de $P$. taeda e $A$. angustifolia exibiram maior retenção a todos os produtos preservantes.

Quanto a perda de massa, independente do tratamento aplicado, a madeira de A. angustifolia foi classificada como resistente ao fungo de podridão branca ( $T$. versicolor). Em contrapartida, o $P$. taeda e o $E$. viminalis foram moderadamente resistentes a este agente.

A madeira de E. viminalis pode ser classificada como resistente ao ataque do fungo $G$. trabeum e altamente resistente quando tratada com óleo de nim. Já as espécies de $A$. angustifolia e $P$. taeda podem ser classificadas como de resistência moderada ao ataque do fungo.

De modo geral, em comparação aos resultados encontrados para as outras espécies, todos os tratamentos foram eficientes para a madeira de $P$. taeda quando em contato ao fungo de podridão parda ( $G$. trabeum).

\section{AGRADECIMENTOS}

À Fundação de Amparo à Pesquisa e Inovação do Estado de Santa Catarina - FAPESC pelo apoio financeiro ao projeto.

\section{REFERÊNCIAS}

AHN SH et al. 2008. Efficacy of wood preservatives formulated from okara with copper and/or boron salts. Journal Wood 
Science 54: 495-501.

AMARAL LS. 2012. Penetração e retenção do preservante em Eucalyptus com diferentes diâmetros. Dissertação (Mestrado em Ciência e Tecnologia da Madeira). Lavras: UFLA. 81p.

ASTM. 2005. American Society for Testing and Materials. Standard test method for accelerated laboratory test of natural decay resistance of wood: ASTM D 2017. West Conshohocken, 5p.

ASTM. 1999. American Society for Testing and Materials. Standard test method for wood preservatives by laboratory soil-block cultures: ASTM D 1413. West Conshohocken: ASTM. 7p.

BALLARIN AW \& PALMA HAL. 2003. Propriedades de resistência e rigidez da madeira juvenil e adulta de Pinus taeda L. Árvore 27: 371-380.

BELTRAME R et al. 2010. Propriedades físico-mecânicas da madeira de Araucaria angustifolia (Bertol.) em três estratos fitossociológicos. Ciência da Madeira 1: 54-69.

BOSSARDI K. 2014. Tall oil e seus subprodutos: alternativas como preservantes para madeira. Tese (Doutorado em Engenharia Mecânica). Guaratinguetá: UNESP. 73p.

CARVALHO SM \& FERREIRA DT. 1990. Santa Bárbara contra vaquinha. Ciência Hoje 11: 65-67.

CASTELLANI A. 1964. The "water cultivation" of pathogenic fungi. Institute of Tropical Medicine 44: 217-220.

DIAS KB \& BARREIROS RM. 2017. Potentiality of tall oil as preservative for wood. Chemical and Biomolecular Engineering 2: 180-183.

DURLO MA. 1991. Tecnologia da madeira: peso específico. Santa Maria: UFSM. 29p. (Série Técnica 8).

FRIGERI JV et al. 2017. Determinação da densidade aparente para as espécies florestais Araucaria angustifolia e Eucalyptus spp. In: Semana de Aperfeiçoamento em Engenharia Florestal da UFPR, Anais... Curitiba: UFPR. 1p.

HYVÖNEN A et al. 2005. Tall oil/water - emulsions as water repellents for Scots pine sapwood. European Journal of Wood and Wood Products 64: 68-73.

IWAKIRI S et al. 2013. Evaluation of the use potential of nine species of genus Eucalyptus for production of veneers and plywood panels. Cerne 19: 263-269.

KLOCK U. 2000. Qualidade da madeira juvenil de Pinus maximinoi H.E. Moore. Tese (Doutorado em Ciências Florestais). Curitiba: UFPR. 291p.

LOPES CSD. 2007. Caracterização da madeira de três espécies de eucalipto para uso em movelaria. Dissertação (Mestrado em Recursos Florestais). Piracicaba: USP. 88p.

MACHADO GO et al. 2006. Preservante natural de madeira para uso na construção civil - óleo de Neem. Minerva 3: 18.

MARTÍNEZ AT et al. 2005. Biodegradation of lignocellulosics: microbial, chimical, and enzymatic aspects of the fungal attack of lignin. International Microbiology 8: 195-204.

MELCHIORETTO D \& ELEOTÉRIO JR. 2003. Caracterização, classificação e comparação da madeira de Pinus patula, $P$. elliottii e $P$. taeda através de suas propriedades físicas e mecânicas. In: Congresso Regional de Iniciação Científica e Tecnológica. Anais... Blumenau: FURB. p.1-5.

MENDES AS \& ALVES MVS. 1988. Degradação da madeira e sua preservação. Brasília: IBDF/DPq-LPF. 56p.

MORESCHI JC. 2014. Biodegradação e preservação da madeira: fatores que afetam a qualidade do tratamento preservativo da madeira maciça. Curitiba: UFPR. 161p. (Material Didático).

PANOV D \& TERZIEV N. 2010. Plant oils as "green" substances for wood protection. In: International Conference on Environmentally-Compatible Forest Products. Anais... Porto: ECOWOOD. p.143-149.

PAES JB et al. 2012. Eficiência dos óleos de nim (Azadirachta indica A. Juss.) e mamona (Ricinus communis L.) na resistência da madeira de sumaúma (Ceiba pentandra (L.) Gaerth) a fungos xilófagos em simuladores de campo. Ciência Florestal 22: 617-624.

PROCITROPICOS. 2014. Programa Cooperativo de Investigación, Desarrollo e Innovación Agrícola para los Trópicos Suramericanos. Compostos Fenólicos. Disponível em: <https://procitropicos.org.br/articulo/artigo-compostos-fenolicosen-portugues/>. Acesso em: 27 set. 2018.

ROLIM MB \& FERREIRA M. 1974. Variação da densidade básica da madeira produzida pela Araucaria angustifolia (BERT.) O. Kuntze em função dos anéis de crescimento. IPEF 9: 47-55.

SALES-CAMPOS $\mathrm{C}$ et al. 2003. Estudo da variabilidade da retenção do preservante CCA tipo A na madeira de Brosimum rubescens Taub. Moraceae - (pau-rainha) uma espécie madeireira da região amazônica. Árvore 27: 845853.

SINGH T \& SINGH AP. 2011. A review on natural products as wood protectant. Wood Science and Technology 46: 851870.

SOARES CHL. 1998. Estudos mecanísticos de degradação de efluentes de indústrias de papel e celulose por fungos basidiomicetos degradadores de madeira. Tese (Doutorado em Química Orgânica). Campinas: UNESP. 120p.

SOUZA RV \& DEMENIGHI AL 2017. Tratamentos preservantes naturais de madeiras de floresta plantada para a construção civil. Mix Sustentável 3: 84-92.

STURION JA et al. 1988. Qualidade da madeira de Eucalyptus viminalis para fins energéticos em função do espaçamento e idade de corte. Colombo: Embrapa. 5p. (Boletim de Pesquisa Florestal 16).

TEIXEIRA JG et al. 2015. Eficiência do óleo de neem e dos resíduos de candeia sobre a inibição do desenvolvimento de fungos xilófagos. Scientia Forestalis 43: 417-426.

TEMIZ A et al. 2008. Decay resistance of wood treated with boric acid and tall oil derivates. Bioresource Technology 99: 2102-2106. 
VIVIAN MA et al. 2012. Qualidade do tratamento preservativo em autoclave para a madeira de Eucalyptus grandis e Eucalyptus cloeziana. Scientia Forestalis 40: 445-453.

WANG SY et al. 2005. Antifungal activities of essential oils and their constituents from indigenous cinnamon (Cinnamomum osmophloeum) leaves against wood decay fungi. Bioresource Technology 96: 813-818. 